\title{
Tabata training: one of the most energetically effective high-intensity intermittent training methods
}

\author{
Izumi Tabata ${ }^{1}$ (1)
}

Received: 3 December 2018 / Accepted: 8 April 2019 / Published online: 19 April 2019

(c) The Physiological Society of Japan and Springer Japan KK, part of Springer Nature 2019

\begin{abstract}
For decades, high-intensity interval/intermittent exercise training methods have been used by elite athletes to improve their performance in sports. One of the most effective training methods, i.e., 'Tabata training,' is reviewed herein from the viewpoint of the energetics of exercise. The prior research describing the metabolic profile and effects of Tabata training is also summarized, with some historical anecdotes.
\end{abstract}

Keywords Aerobic $\cdot$ Anaerobic $\cdot$ Intermittent exercise $\cdot$ Sports activity $\cdot$ High-intensity

\section{Introduction}

Interval training has been used for decades by elite athletes seeking to improve their sports performance [1]. The interval training method known as Fartlek training was invented by the Swedish coach Gösta Holmér in the 1930s [2]. Dr. Woldemar Gershler also formalized a structured system of interval training in Germany in the 1930s [3]. Interval training was popularized by the Czech runner Emil Zátopek, who won gold medals in the 5000- and 10,000-m races as well as the marathon at the Helsinki Olympic games in 1952 [4].

Thus, interval training itself is not new, and it was extensively investigated during the 1970s. The effects of highintensity interval training on the human body's aerobic energy-releasing system were thoroughly examined by Edward Fox $[1,5]$. He showed that the improvement of the body's maximal oxygen uptake $\left(\mathrm{VO}_{2} \mathrm{max}\right)$ after high-intensity interval training is linearly related to the oxygen demand (expressed as $\% \mathrm{VO}_{2} \mathrm{max}$ ) of the high-intensity interval training, indicating that exercise intensity is a key factor for the improvement of the body's maximal aerobic power after high-intensity interval training [5-7]. Further, Fox showed that the improvement of the $\mathrm{VO}_{2}$ max after high-intensity interval training performed 2 days/week is not different from

Izumi Tabata

tabatai@fc.ritsuei.ac.jp

1 Faculty of Health and Sport Science, Ritsumeikan University, 1-1-1 Noji-Higashi, Kusatsu City, Shiga 525-8577, Japan that achieved by training with this regimen 4 days/week [5, 7]. Since 3 days/week is the recommended frequency of training to improve the $\mathrm{VO}_{2}$ max by conventional moderateintensity exercise training, it is apparent that high-intensity interval training is a potent stimulus for improving one's maximal aerobic power [8]. Thus, high-intensity exercises and training have been used by elite athletes to improve their performance in sports, as such high-intensity exercise was shown to extensively recruit the aerobic energy-supplying system, resulting in the increased maximal oxygen uptake that is the most reliable factor for endurance.

New and important information about high-intensity interval training became available in 1980 - i.e., the anaerobic profile of high-intensity interval training. There had been a lack of quantification of anaerobic energy during high-intensity exercise before the 1980s, when the late Lars Hermansen proposed a method $[9,10]$ for quantifying the anaerobic energy release that uses the accumulated oxygen deficit, which was first introduced by Krogh and Lindhard in 1920 [11]. The anaerobic energy release [which is another aspect of the energy supply for resynthesizing the adenosine triphosphate (ATP) consumed during exercise, especially maximal- to supramaximal-intensity exercises] had not been quantified. 'Accumulated oxygen deficit' is defined as the difference between the accumulated oxygen demand and the accumulated oxygen uptake measured during exercise. This principle was further used to estimate the accumulated oxygen deficit during a high-intensity intermittent exercise [12] by the first author of the original 1996 paper describing Tabata training [12] (Izumi Tabata), who had studied at 
the Institute of Muscle Physiology in Oslo, Norway under the supervision of Dr. Hermansen and learned the principle directly from him.

\section{Nomenclature}

Before this review is considered, the nomenclature regarding Tabata training and high-intensity interval/intermittent training should be established. Tabata training is defined as training at the intensity that exhausts subjects during the 7 th or 8 th sets of 20 -s bicycle exercise bouts with a 10 -s rest between the exercise bouts. This exercise/training was originally developed for bicycling exercise [12, 13]. Regarding similar protocol training that uses other types of exercise including running and various body-weight-bearing exercises (e.g., burpees and squat jumps), the published evidence of their metabolic profiles and effects on both $\mathrm{VO}_{2}$ max and the maximal accumulated oxygen deficit (MAOD) is insufficient. In this review, we therefore focus on Tabata training, defined as bicycle training at the intensity that exhausts subjects during seven or eight sets of 20-s bicycle exercise bouts with a 10-s rest between the bouts.

At more than 20 years after the publication of the original study [12,13], the exercise intensity has not been emphasized; only the procedure of the training has been featured, especially among general exercisers. For example, following such a protocol (eight sets of a 20 -s exercise with a 10-s rest between the exercise bouts) using walking as the exercise can be expected to result in no improvement of the $\mathrm{VO}_{2} \max$. Only training adopting the protocol with an exercise intensity that exhausts the subject after 7-8 sets of the 20-s exercise bout with a 10-s rest between the exercise bouts (i.e., Tabata training) elevates both the $\mathrm{VO}_{2}$ max and the MAOD to the extent that was reported by the original investigation.

Such increases in the two energy-releasing systems (i.e., the aerobic and anaerobic energy-releasing systems) cannot be obtained by walking, the exercise intensity of which is estimated as $<30 \% V \mathrm{O}_{2}$, in Tabata protocol. Therefore, the term 'Tabata training' emphasizing not only the procedure but also the exercise intensity that exhausts the subject after 7-8 sets of the exercise should be used for the name of the training, and this term will be used hereafter in this review $[12,13]$.

\section{Interval or intermittent?}

In a popular method of interval training, an individual exercises at low intensity between bouts of high-intensity exercise [1]. In contrast, intermittent training (including Tabata training $[12,13])$, exercisers completely stop the exercise and rest for a while. Training that involves such a 'complete stop' period is thus called 'intermittent' training $[14,15]$. Intermittent training and interval training thus differ significantly, and it is important to keep in mind that Tabata training is an intermittent-exercise training method.

\section{HIIT, SIT, or?}

Tabata training has been considered one of the high-intensity 'interval or intermittent' training (HIIT) methods, which have varied considerably in terms of the characteristics of the training exercise, i.e., the exercise mode, intensity, and durations of exercise and rest. Weston et al. defined HIIT as 'near maximal' (in other words, 'submaximal') effort generally performed at an intensity that elicits $>80 \%$ (often $85-95 \%$ ) of the maximal heart rate [16]. Thompson suggested a broader definition of HIIT in which HIIT typically involves short bursts of high-intensity exercise followed by a short period of rest or recovery and typically takes $<30$ min to perform [17].

In contrast, sprint interval training (SIT) is characterized by efforts performed at intensities equal to or greater than the pace that would elicit a $V \mathrm{O}_{2}$ peak, including 'allout' or 'supramaximal' efforts [16]. The word 'sprint' implies moving as fast as possible from the start of an exercise [18], with an eventual decline in speed and/or a discontinuation of the exercise. In contrast, in the original and authentic Tabata training protocol, the exercise intensity is constant (i.e., $170 \% V_{2}$ max) from the first to the last session of the exercise. Using the word 'sprint' to describe Tabata training exercise is therefore not accurate.

In exercise physiology, the intensity of a specific exercise has been defined relative to the $V \mathrm{O}_{2}$ max as 'submaximal,' 'maximal,' and 'supramaximal' when the oxygen demand is less than, equal to, and greater than the $V \mathrm{O}_{2} \max$, respectively. Since the oxygen demand for Tabata training is higher than the $V \mathrm{O}_{2} \max$ (i.e., $170 \% \mathrm{VO}_{2} \max$ ), the original Tabata training is 'supramaximal intensity intermittent training.' In terms of the exercise:recovery ratio, Tabata training is different from other SITs, as Sloth et al. [19] defined SIT as a protocol that includes duration of bouts: 10-60 s, intensity: maximal, "all-out", volume: $\geq 12$ repetitions, recovery: $\geq 5$ times the duration of work, and Gist et al. [20] defined it as intensity: "all-out", "supramaximal", "maximal" or " $\geq V \mathrm{O}_{2} \max$ ", SIT work:rest ratio of $30-\mathrm{s}: 4-\mathrm{min}$ (rest interval of $3-5 \mathrm{~min}$ ). Tabata training is thus not SIT in terms of the classical terminology of SIT. Tabata training is an original and unique training method that can be described by either the classic but familiar term 'interval training' or the modern and "cool" term 'HIIT,' which includes a variety of training methods using intermittent/interval high-intensity exercise. 


\section{The metabolic profile of Tabata training exercise}

The endurance profile of most exercises and sports is thought to depend on the amount of energy output per unit of time. Since the energy output (i.e., the ATP consumption/resynthesis) is equal to the energy supply from the body's aerobic and anaerobic energy-supplying systems, it well recognized that increasing the functioning of the two energy-supplying systems by physical training is an optimal way to enhance endurance performance [21, 22]. Endurance training elevates the maximal aerobic power [23-25], whereas sprint training using very brief exercise at high intensity increases the body's anaerobic capacity measured as the MAOD [26]. For most physical properties, the more demanding the training is, the greater the improvement of the property will be, and it is thus necessary to measure the energy supply from both the aerobic and anaerobic energy-supplying systems during exercise in order to evaluate the training's efficacy. The aerobic-energy release is well quantified by measuring the $V \mathrm{O}_{2}$. Therefore, by measuring the oxygen uptake and comparing that value with the subject's $V \mathrm{O}_{2} \max$, the demand of the exercise/training on the aerobic energy-releasing system can be evaluated.

Since, at a submaximal exercise intensity, the energy from the anaerobic energy-releasing system is supplied only at the beginning of the exercise, the relative contribution of this system is low [27]. In contrast, the anaerobic energyreleasing system contributes significantly to the total energy demand by 35,53 , and $70 \%$ during exhaustive exercises at the supramaximal intensity of 119,146 , and $186 \% \mathrm{VO}_{2}$ max, respectively [28]. As evaluating stress of a specific exercise on aerobic energy-releasing system as a training, stress on the anaerobic energy-releasing system can be evaluated by comparing the accumulated oxygen deficit during an exercise to the anaerobic capacity (i.e., the MAOD). However, high-intensity intermittent exercise, which is often used as a training method, had not been evaluated using the same methods until we assessed the energy release from both the aerobic and anaerobic energy-releasing systems during two different intermittent exercise protocols [12], which are used by some of the top Japanese speed skaters. We analyzed the two training protocols for the following reasons.

The two training protocols were introduced by Kouichi Irisawa, who was a head coach of the Japanese Speed Skating Team in the 1980s. Mr. Irisawa (who was sent by the Japan Sport Association as a visiting guest to the Norwegian Skate Federation) and the first author (I. Tabata) of the original investigation of Tabata training $[12,13]$ stayed in the same dormitory at the University of Oslo for most of 1984. After returning to Japan, Tabata joined the Japan National Speed Skating Team led by Mr. Irisawa as the fitness coach for the Albertville Olympics to be held in 1992. After discussion during the National team's training camp in 1989 in Maebashi in Japan's Gunma Prefecture about the selection of the best training method, the two training methods developed by Mr. Irisawa and used by top Japanese skaters were compared in a study [12] conducted at a laboratory at the National Institute of Fitness and Sport in Kanoya, which is located in the southernmost region of Japan.

The study compared two intermittent bicycle exercise (IE) protocols (IE1 and IE2) [12]. The results of that investigation showed that the accumulated oxygen deficit during the exhaustive intermittent exercise of IE1 (exercise intensity: approx. $170 \% \mathrm{VO}_{2} \max , 7-8$ bouts of 20 -s exercise with a 10-s rest between bouts) equaled the anaerobic capacity (i.e., MAOD), and thus the IE1 protocol seemed to stress the anaerobic energy system maximally (Fig. 1). In addition, the IE1 protocol recruited the oxygen delivery system maximally since the oxygen uptake measured during the last part of the IE1 protocol was not different from the $V_{2} \max$ of the subjects (Fig. 2).

In contrast, neither the anaerobic system nor the aerobic system seemed to be fully stressed during the exhaustive intermittent exercise of the IE2 protocol (exercise intensity: approx. 200\% $\mathrm{VO}_{2} \max , 3-4$ bouts of 30-s exercise with a 2 -min rest between bouts). These results demonstrated that, for the purpose of improving both the anaerobic and aerobic energy-releasing systems, the IE1 protocol was superior to the IE2 protocol. The IE1 protocol was also confirmed to stimulate both the aerobic and anaerobic energy-releasing systems maximally. Since the human body has only these two energy-supplying systems, the IE1 protocol can be

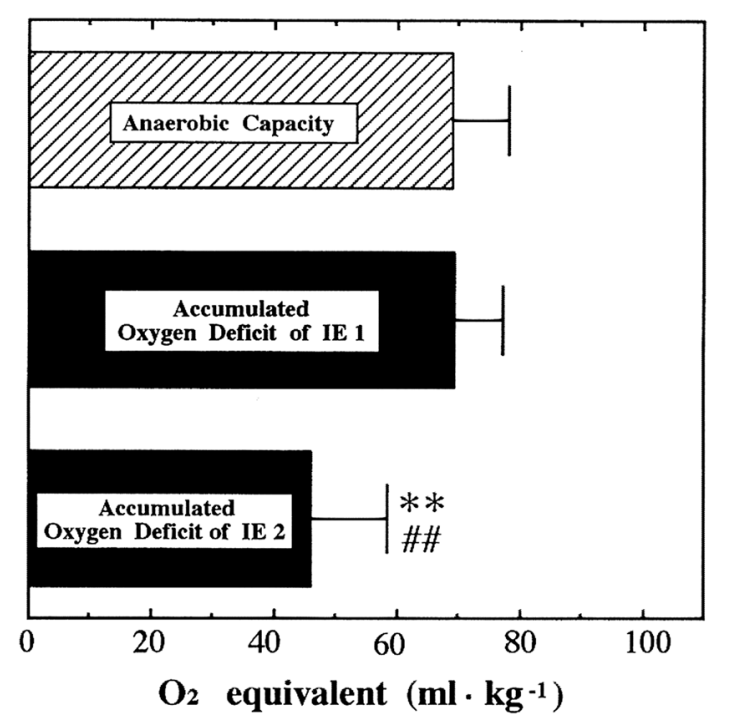

Fig. 1 Accumulated oxygen deficit during the intermittent exercise (IE) 1 protocol (Tabata training) and the IE2 protocol and the anaerobic capacity, i.e., the maximal accumulated oxygen deficit (MAOD) [12]. ${ }^{* *} p<0.01$ vs. the anaerobic capacity (MAOD). ${ }^{\# \#} p<0.01$ vs. the accumulated oxygen deficit in the IE1 protocol 




Fig. 2 Peak oxygen uptake during the last $10 \mathrm{~s}$ of the IE1 (Tabata training exercise) and IE2 protocols, and the maximal oxygen uptake [12]. ${ }^{* *} p<0.01$ vs. the maximal oxygen uptake. ${ }^{\#} p<0.05$ vs. the peak oxygen uptake during the last $10 \mathrm{~s}$ of the IE1 protocol

regarded is one of the most energetically effective exercise training protocols for maximally improving both the aerobic and anaerobic energy-supplying systems.
As mentioned above, since the IE1 training protocol emerged during discussions between a top coach who had an instinct for develop new training methods based on interactions with athletes and an exercise physiologist who was good at scientifically analyzing the characteristics of exercise, Tabata training was both clinical (practical)- and bedside (rink-side or gym-side)-initiated training. After the above-described results were reported to Mr. Irisawa, he stopped using the IE2 protocol as part of the training menu for the skaters and concentrated on the use of the IE1 protocol (i.e., Tabata training).

For anaerobic energy quantification, especially during 0-10 min of exhaustive exercise of which the exercise intensity is above the $\mathrm{VO}_{2} \mathrm{max}$, an estimation of the oxygen demand for such exercise is required. The oxygen demand is a value $(\mathrm{L} / \mathrm{min}$, or $\mathrm{ml} / \mathrm{kg} / \mathrm{min})$ representing the oxygen that the body needs for a specific exercise at a specific intensity (Fig. 3). For a submaximal-intensity exercise, the oxygen demand for a specific exercise is measured as the oxygen uptake during the exercise. For a submaximalintensity exercise, the oxygen demand of which the oxygen uptake has not been measured, the oxygen demand can be estimated quite accurately by interpolation from the linear relationship between the exercise intensity $(W)[12,13]$
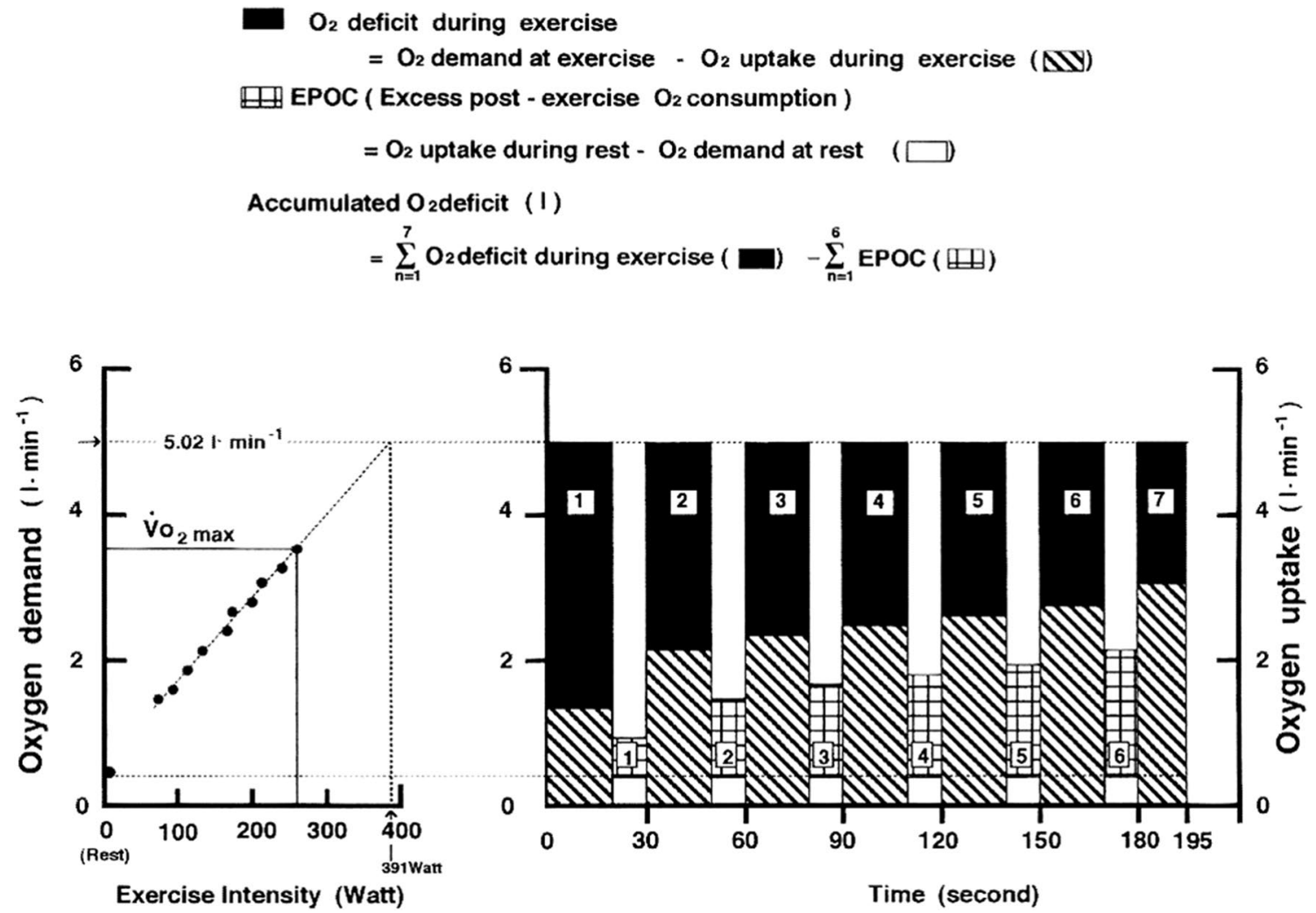

Fig. 3 Principle used to calculate the accumulated oxygen deficit for high-intensity intermittent exercise [12] 
(Fig. 3) and the measured oxygen uptake, in the same way as that used for running $(\mathrm{m} / \mathrm{min})[10]$.

This relationship was established by measuring the oxygen uptake at 6-9 different intensities of 10-min exercises. This time-consuming procedure, which need at least 3 days of testing, has been also used to measure the $V \mathrm{O}_{2} \max$ in Scandinavian countries. Without establishing a linear relationship ( $r>0.99)$ between the oxygen uptake and the exercise intensity at a submaximal range, the leveling off of the oxygen uptake in response to increased exercise intensity, which is the only criterion for measuring the maximal oxygen uptake [29], cannot be confirmed. Without this leveling off, the measurement is regarded not as the $V_{2} \mathrm{O}_{2}$ max but rather as the peak $V \mathrm{O}_{2}$. The measurement of the oxygen uptake at ten different intensities of 4-min exercises could be used to establish the relationship between the oxygen uptake and work rate at a submaximal level [30]. Since the work rate during swimming is theoretically related to [swimming speed $\left.(\mathrm{m} / \mathrm{s})^{3}\right]$, a linear relationship between exercise intensity and oxygen uptake is established by the relationship between the exercise intensity [swimming speed $(\mathrm{m} / \mathrm{s})^{3}$ ] and the oxygen uptake [31].

In contract to submaximal-intensity exercise, the oxygen uptake of a supramaximal-intensity exercise cannot be measured; if the oxygen uptake at such high intensity is higher than the $V \mathrm{O}_{2} \max$, it is the $V \mathrm{O}_{2} \max$ of the subject. Therefore, in terms of expressing the intensity of a supramaximal-intensity exercise relative to the $V \mathrm{O}_{2}$ max, similar to the exercise intensity of $170 \% \mathrm{VO}_{2} \max$ for the IE1 protocol (Tabata training) [12], the oxygen demand (not the oxygen uptake) is used, and the oxygen demand is estimated by extrapolating the linear relationship between exercise intensities at submaximal levels and the measured oxygen uptake (Fig. 3). For example, the oxygen demand of $170 \% \mathrm{VO}_{2}$ max for bicycling is calculated as 1.70 times the $\mathrm{VO}_{2}$ max measured for bicycling.

It should be noted that, for estimating oxygen demand at supramaximal intensity, the relationship between exercise intensity (the work rate) and the submaximal level oxygen uptake measured by an incremental test [e.g., a graded exertion test (GXT)] [32] should not be used. The oxygen uptake at a specific exercise intensity measured by an incremental test procedure, which normally allots an identical time (1-2 min) for each exercise intensity, does not necessarily represents the oxygen uptake or oxygen demand, which is balanced with energy for re-synthesizing the ATP consumed during exercise at the specific intensity. This is because the time necessary for the oxygen uptake to reach the steady state (which is defined as the oxygen uptake/demand balance of the specific exercise) differs based on the exercise intensity. The time necessary for the oxygen uptake to reach the steady state of oxygen consumption at a higher exercise intensity is longer than that at a lower exercise intensity [27].
Therefore, the original investigation of anaerobic capacity (MAOD) measured oxygen uptake during 6-9 bouts of 10-min constant-intensity exercises whose intensity ranges from approx. $30 \%$ to $85 \%$ of the $\mathrm{VO}_{2} \max$ [10].

The original investigation $[10,12,13]$ used such a timeconsuming test for the following reasons: (1) to measure the $\mathrm{VO}_{2}$ max correctly by ascertaining the leveling-off, and (2) to correctly estimate the oxygen demand at supramaximal intensity for calculating the accumulated oxygen deficit, which is defined as the difference between the total accumulated oxygen demand (L), [i.e., the estimated oxygen demand $(\mathrm{L} / \mathrm{min}) \times$ the exercise time $(\mathrm{min})]$ and the accumulated oxygen uptake $(\mathrm{L})$ measured during the exercise.

\section{The effects of Tabata training on the body's aerobic and anaerobic energy-releasing systems}

Six weeks of training using the IE1 protocol, which was later named Tabata training, was found to increase the MAOD by $28.0 \pm 19.4 \%$ (Fig. 4) and the $V_{2}$ max by $15.0 \pm 4.7 \%$ (Fig. 5) [13]. This training consisted of 4 days/week of exhaustive IE1 exercises (7-8 sets to exhaustion) and 1 day/ week of $30 \mathrm{~min}$ of continuous exercise at $70 \% \mathrm{VO}_{2} \max$ and four subsequent sets of the IE1 protocol, which was not exhaustive. The results of that study suggested that this high-intensity intermittent exercise is a very effective tool to improve sports-related physical fitness. Since this training improved the subjects' $\mathrm{VO}_{2} \max$ and MAOD during the training period, the training subjects became able to bike $>8$ sets of the 20-s exercise at the first-prescribed intensity for the training. At that time point, the intensity (i.e., the work rate for bicycling) was increased by 11 watts so that the exercise exhausted the subjects within 7-8 sets of 20-s exercise. The important thing is that, during this training period, the

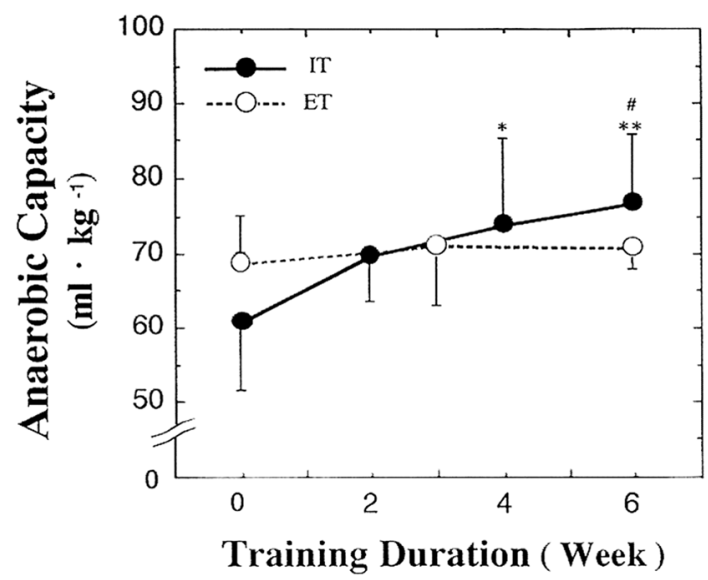

Fig. 4 Effect of endurance training (ET) and intermittent training (IT; Tabata training) on the anaerobic capacity, i.e., the maximal accumulated oxygen deficit (MAOD) [13]. ${ }^{*} p<0.05,{ }^{*} p<0.01$ increase vs. the pretraining value. ${ }^{\#} p<0.05$ increase vs. the 2 -week value 


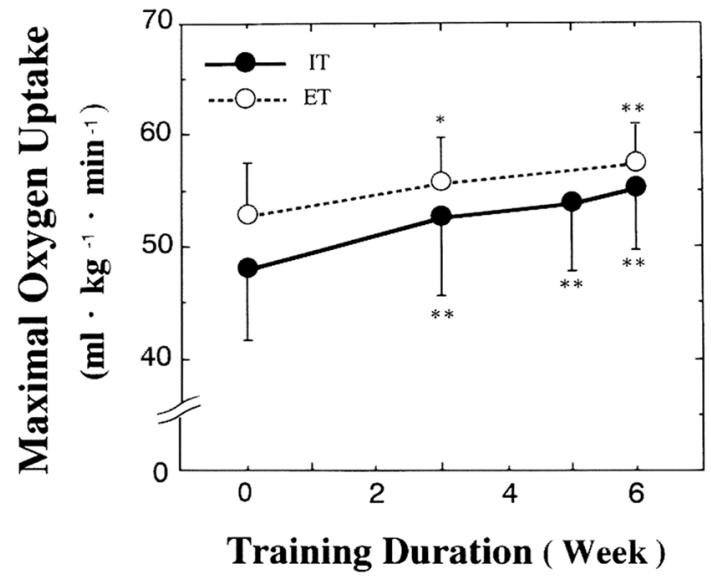

Fig. 5 Effects of ET and IT (Tabata training) on the maximal oxygen uptake [13]. ${ }^{*} p<0.05,{ }^{*} p<0.01$ increase vs. the pretraining value

exercise intensity that exhausts the subject within $7-8$ sets of the 20 -s exercise should be prescribed during the entire training period.

It is well known that there is a site specificity of training and its effects. As an example of site specificity, only the functioning of the lower-leg muscles is improved by exercise using the lower legs, e.g., squats. No effects are expected to be found in the functioning of the arm muscles. There is also specificity regarding energy-releasing systems. Anaerobic training improves the body's anaerobic capacity measured as the MAOD, whereas aerobic training elevates the aerobic capacity measured as the $\mathrm{VO}_{2}$ max. For example, Medb $\varnothing$ and Burgers [26] reported that training with eight bouts of 20 -s running exercise at $165 \% \mathrm{VO}_{2}$ max with a $4.5-5.0$-min pause between the bouts increased the subjects' MAOD by approx. $10 \%$.

Aerobic training consisting of 1-h prolonged bicycle exercise at $70 \% \mathrm{VO}_{2} \mathrm{max}$ increased the $\mathrm{VO}_{2} \mathrm{max}$ without effects on the MAOD [13]. During the last session of the IE1 protocol, the oxygen uptake reached the $\mathrm{VO}_{2} \mathrm{max}$ (which is a measure of aerobic capacity), and the accumulated oxygen deficit of the training exercise amounted to the MAOD (which is a measure of anaerobic capacity). The magnitude of the effects of specific training on a specific aspect of fitness may depend on the extent to which the training stresses the subject's fitness. Since humans have only two energyreleasing systems and the IE1 protocol stresses both systems maximally, training using the IE1 protocol can be regarded as one of the ultimate aerobic and anaerobic training methods. Consequently, according to the system specificity of training and its effects on energy release, Tabata training enhances both the $\mathrm{VO}_{2}$ max and the MAOD.

Since the intensity of the IE1 protocol is quite high compared to other types of so-called 'aerobic exercise,' training using the IE1 protocol looks like 'anaerobic training.'
If this is so, and if the effect is expected according to the specificity of energy release, training that uses the IE1 protocol is not expected to improve the $V_{2} \max$. However, the oxygen uptake at the end of training using the IE1 protocol reaches the $V \mathrm{O}_{2} \max$. This result, i.e., that the $\mathrm{VO}_{2} \max$ was increased by the training, can be explained by the specificity of training and training effects regarding energy release. One may think it strange that the study's authors [12] measured oxygen uptake during the IE1 that appears to be anaerobic exercise. This was simply because, for the calculation of the oxygen deficit value needed for the determination of the anaerobic energy release of the training, the oxygen uptake had to be measured.

After the publication of these two papers [12, 13], training using the IE1 protocol began to be referred to as the 'Tabata protocol,' 'Tabata interval training,' or 'Tabata-style training,' and these terms began to be used by many people, including both sport-oriented athletes and health-oriented non-athletes. Further research on 'Tabata' or 'Tabata-style' exercise training was then conducted. For example, Foster et al. elegantly reproduced the effect of 8 weeks of Tabata training on aerobic energy-releasing system (18\% increase in the $\mathrm{VO}_{2} \max$ ) [33].

As shown by Foster et al. [33], the Tabata training method is quite demanding for ordinary young adults. The use of this training method needs (1) high motivation of elite athletes who want to elevate both their aerobic and anaerobic energy-releasing systems, and (2) convincing instruction to the athletes from coaches who fully understand the scientific evidence regarding this method.

\section{Different high-intensity intermittent exercise protocols}

In their efforts to devise a more efficient training method than Tabata training, Kouzaki and Tabata compared several other high-intensity intermittent bicycle exercise protocols in terms of the recruitment of the aerobic and anaerobic energy-releasing systems [34]. Their study revealed that the most demanding protocol was the 'IDE200' protocol, which used the intensity $200 \% V_{2}$ max for the first and second sets, $180 \% \mathrm{VO}_{2}$ max for the third and fourth sets, and $160 \%$ $\mathrm{VO}_{2}$ max for the fifth and sixth sets of 20 -s exercise bouts separated by 10 -s rests. This was because the oxygen deficit during the IDE200 protocol and the oxygen uptake during the last part of the protocol were not significantly different from those observed during the IE170 protocol (i.e., Tabata training).

In addition, the peak lactate concentration after the IDE200 protocol was significantly higher than that observed after the IE170 protocol. The reasons for the comparison of the IDE200 protocol with Tabata training (IE170) were as follows. Even during such high-intensity exercise, which 
appears to be 'anaerobic' exercise, the oxygen uptake during the first $20 \mathrm{~s}$ is related to the oxygen demand of the exercise, which is expressed as the $\% V_{2} \max$ (Kouzaki and Tabata, unpublished observation), and the higher the speed of the recruitment of aerobic energy during the beginning of the high-intensity exercise, the higher the oxygen uptake was during the last phase of the exercise.

Secondly, the oxygen deficit that accumulated during the 30 -s exhaustive exercise $\left(200 \% \mathrm{VO}_{2} \max \right)$ does not reach the maximal oxygen deficit $[7,18]$, suggesting that such highintensity exercise to exhaustion does not recruit anaerobic energy maximally. To ensure a full recruitment of anaerobic energy release, a somewhat low intensity is required. Therefore, the exercise intensity used for the last two bouts was $160 \% \mathrm{VO}_{2} \mathrm{max}$, which corresponds to the intensity that exhausts the subjects at approx. 1-2 min and which was shown to maximally stimulate the anaerobic energy-releasing system.

After an 8-week training using the IDE200 protocol 5 days/week, the subjects' MAOD was increased significantly (by $32 \%$ ), and the $\mathrm{VO}_{2}$ max was also elevated significantly (by $14 \%$ ) [34].

Ogita et al. reported that a higher-intensity (approx. 250\% $V_{\mathrm{O}_{2}} \max$ ) and shorter-duration (five 5 -s exercise bouts with a 10-s rest between bouts) intermittent swimming training protocol performed for 4 weeks improved the swimmers' MAOD and $\mathrm{VO}_{2}$ max by $22 \%$ and $5 \%$, respectively [35].

\section{The effects of Tabata training and resistance training on the MAOD}

Principally, the MAOD is proportional to the muscle volume. This is because the greater the muscle volume, the more creatine phosphate is available in whole muscle and the more lactate accumulates; creatine phosphate and lactate are the bases of the alactic and lactic energy release that respectively comprise anaerobic energy release. Changes in the MAOD after resistance training that enlarges muscle volume were thus investigated by Hirai and Tabata [36]. In that study, the training consisted of a high-intensity intermittent exercise training (IT; in this case, Tabata training) for 6 weeks and IT plus resistance training (RT) for the subsequent 6 weeks. During the IT-alone period, the subjects trained using IT on 5 days/week. During the IT + RT period, they trained using IT on 3 days/week and RT for 3 days/week. The IT increased the subjects' MAOD by 17\% (Fig. 6). The RT consisted of (a): four sets of 12 repetitions of squat and leg curl exercises at 12 repetition max (RM) with a 30-s rest between each set, and (b) two sets of maximal bouts of the same exercise with a load of 90,80 , and $70 \%$ of one RM.

After the IT and RT period, the subject lifted a barbell mass 12 times (12 RM) for squat was increased by

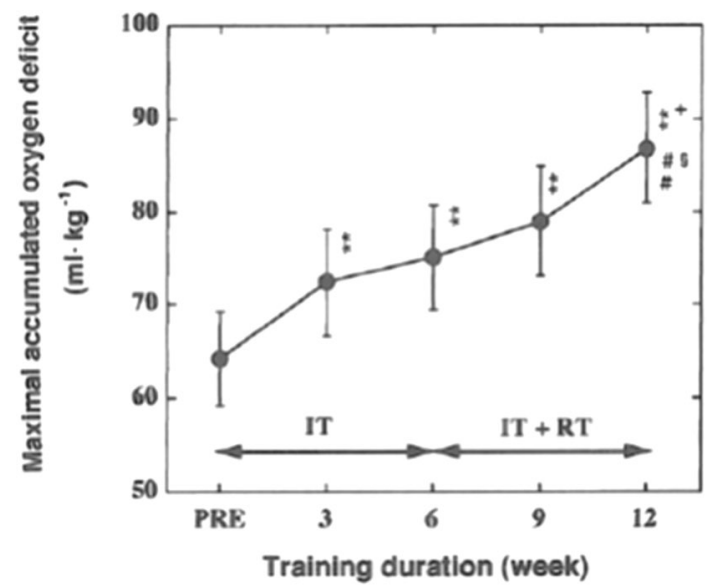

Fig. 6 Effects of IT and resistance training (RT) on the MAOD [36]. ${ }^{*} p<0.05,{ }^{* *} p<0.01$ vs. the pre-training value. ${ }^{+} p<0.05,{ }^{++} p<0.01$ vs. the 3 -week value. ${ }^{\#} p<0.05,{ }^{\#} p<0.01$ vs. the 6 -week value. ${ }_{p} p<0.05$ vs. the 9 -week value

$108 \pm 8 \%$. The IT + RT further increased the subjects' MAOD values, suggesting that an increase in muscle volume as a result of resistance training is effective for increasing the MAOD [36]. However, Minahan and Wood reported that resistance training did not affect the MAOD [37]. It is thus not known whether the increase in the MAOD obtained with an IT + RT regimen is attributable to combined effects of resistance training and HIIT or just to HIIT. Further research can be expected to elucidate this issue.

During the IT-alone period, both the maximal power during the Wingate test, and the circumference $(\mathrm{cm})$ of thigh muscle were not changed. However, after the IT and RT period, the maximal power was significantly increased by $10 \pm 3 \%(p<0.05)$ with a significant increase in the thigh muscle circumference $(3 \pm 1 \%, p<0.01)$ [36]. These results may indicate that (1) Tabata training itself does not affect anaerobic power, and (2) an increase in muscle mass is necessary to induce an increase in anaerobic power.

In the above-described study by Hirai and Tabata [36], the $\mathrm{VO}_{2}$ max increased during the IT period $(11 \pm 2 \%)$, whereas no significant change was observed during the IT + RT period (Fig. 7). This result may indicate that, in terms of high-intensity intermittent training, a different strategy is necessary for further improvement in the aerobic energy-releasing system. Hickson et al. demonstrated that simultaneous training for strength and endurance will result in a reduced capacity to develop strength, but will not affect the magnitude of the increase in the $V \mathrm{O}_{2} \max$ [38]. Studies that eliminate the interference of one type of fitness over another type should be devised to address this issue. 


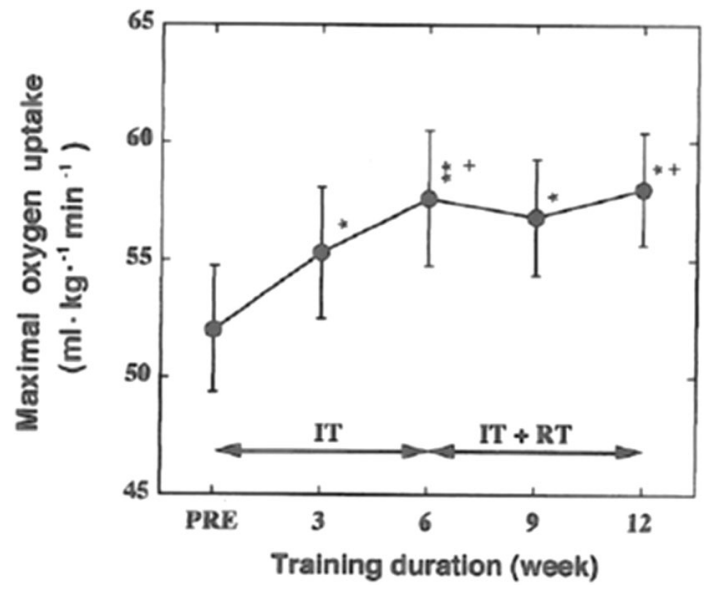

Fig. 7 Effects of the IT and RT on the maximal oxygen uptake [36]. ${ }^{*} p<0.05,{ }^{*} p<<0.01$ increase vs. the pretraining value. ${ }^{+} p<0.05$, ${ }^{++} p<0.01$ vs. the 3 -week value

\section{Metabolic changes in muscle after Tabata training}

Changes in the enzyme activity/content in skeletal muscles recruited during exercise training were studied after Bergstrom introduced the needle biopsy technique in 1962 [39]. Specific training induces increased expressions of proteins that have specific physiological functions in skeletal muscles recruited by training. For example, aerobic training increases the enzyme activities of citrate synthase (CS, which is a key enzyme for oxidative metabolism [40]), and high-intensity exercise training increases glycogen phosphorylase and phosphofructokinase (PFK), which are possible rate-limiting enzymes of anaerobic metabolism [41, 42]. Sprint training with a very short duration of exercise (i.e., 5-s running) without changes in the $V \mathrm{O}_{2}$ max elevated the enzyme activity of myokinase [43].

Skeletal muscle adaptation to Tabata training was recently reported [44]. After 6 weeks of Tabata training, the enzyme activities of CS and PFK were significantly increased (Fig. 8), indicating that the training may have enhanced protein expressions, possibly limiting both the aerobic and anaerobic energy-releasing systems, suggesting that in terms of the two energy-releasing systems, peripheral adaptations occurred after the Tabata training. These elevated enzyme activities may have contributed to the increases in the $\mathrm{VO}_{2} \max (9.2 \%)$ and MAOD (20.9\%).

In addition, along with an enhanced expression of fatty acid oxidative enzyme activity [45], the protein expression of peroxisome proliferator-activated receptor- $\gamma$ coactivator-1 $\alpha(\mathrm{PGC} 1 \alpha)$ was found to be enhanced after rats performed a Tabata training model [46]. Those results suggested that dozens of proteins that are known to be increased by this transcriptional coactivator may be increased after Tabata training [47].

The swimming protocol used for the above-mentioned rat study consists of 14 bouts of intermittent 20 -s swimming with a 10-s rest between the exercise bouts while the rat bears a weight equivalent to $14 \%$ of its body weight [46]. Since it was not feasible for rats to run intermittently at a high intensity (speed) on a treadmill, swimming was introduced to model Tabata training for rats. Because the oxygen uptake during the high-intensity intermittent swimming was not measured in that investigation, the precise percentage of the rats' maximal oxygen uptake during the swimming was not known. The reason that this protocol has been used in previous studies is that the protocol was shown, by trial and error, to raise the blood lactate concentration to levels that were similar to those measured for humans and to elevate the enzyme activity of citrate synthase, which is an oxidative enzyme in mitochondria [48].

Indeed, the above-cited clinical study [44] demonstrated that 79 genes including genes involved in glucose metabolism, the mitochondria membrane, the extracellular matrix, and angiogenesis were increased in skeletal muscle by training. A proteomics analysis of rat muscles after
Fig. 8 Effects of Tabata training on the enzyme activities of phosphofructokinase (PFK) and citrate synthase (CS) [44] (a)

( $\mu \mathrm{mol} / \mathrm{min} / \mathrm{g}$ tissue)

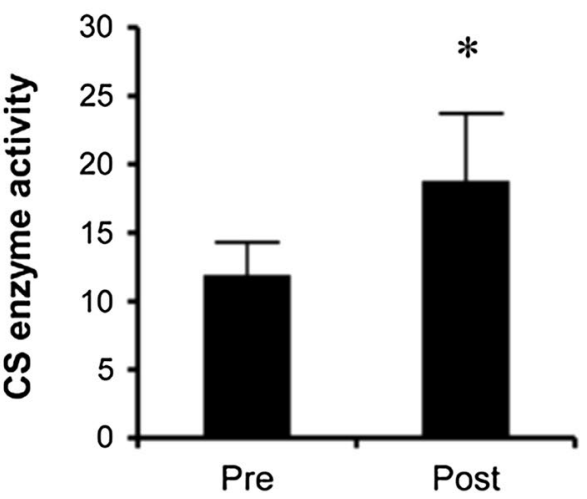

(b) ( $\mu \mathrm{mol} / \mathrm{min} / \mathrm{g}$ tissue)

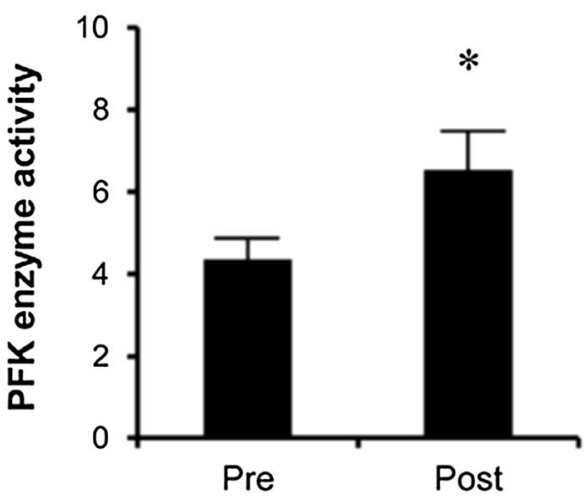


Tabata-model training revealed that the protein expression of glycogen phosphorylase (the first enzyme of glycogenolysis) was increased [49]. It is well documented that low-volume interval training increases skeletal muscle oxidative enzymes [50]. Robinson et al. [51] reported that moderate-intensity HIIT affects the transcription of a large number of proteins in humans.

In terms of the increase in the $\mathrm{VO}_{2}$ max after HIIT, there has been some disagreement regarding whether the main location of the occurrence of adaptation is central (cardiorespiratory: cardiac output) or peripheral (skeletal muscle: metabolic enzymes). The improvement in the $\mathrm{VO}_{2}$ max after specific training may be due to both central and peripheral factors, which correspond to increased cardiac output and oxygen extraction and/or oxygen consumption in working skeletal muscle. These factors can be further explained by increased maximal cardiac output/stroke volume of heart and increased oxidative enzyme activity in skeletal muscle, respectively. Since the increase in the $\mathrm{VO}_{2}$ max after HIIT including Tabata training is very rapid (e.g., 2-3 weeks) $[13,36]$ and changes in the morphology of the heart are not expected during such a short time period, the increase in the $V \mathrm{O}_{2}$ max after Tabata training could be attributed to peripheral factors.

However, Burgomaster et al. [52] reported that after 2 weeks of SIT training, the subjects' $V \mathrm{O}_{2}$ peak did not increase but the activity of CS was enhanced by $38 \%$, suggesting that changes in peripheral factors do not necessarily induce increase in $V \mathrm{O}_{2}$ peak. Daussin et al. suggested that adaptation after interval training occurs both centrally and peripherally [53]. Macpherson et al. reported that 6-week run sprint interval training improved their subjects' aerobic performance but not maximal cardiac output [54], whereas another research group reported that 6-week high-intensity interval training increased their subjects' cardiac output and $\mathrm{VO}_{2} \max$ [55]. The authors of the latter study attributed the initial increase in cardiac output after the early phase of the high-intensity interval training to plasma volume expansion [55], which was apt to occur in their sedentary subjects with lower $\mathrm{VO}_{2}$ max values compared to those of the recreationally active subjects of the Macpherson et al. study. Further research is necessary to address these findings.

In the original Tabata et al. study in 1996 [13], the subjects executed Tabata training exercise for 4 days and non-exhaustive sessions of exercise at $170 \% \mathrm{VO}_{2}$ max after 30 min of exercise at $70 \% \mathrm{VO}_{2}$ max on Wednesday as a break. However, as Fox et al. reported, the frequency of $2 \times /$ week appears to be enough for Tabata training to induce the adaptation of the aerobic energy-releasing system [5, 7]. In terms of peripheral adaptation, the expression of PGC1 $\alpha$, which is a potent transcriptional coactivator for enzymes involved in oxidative metabolism, was maintained for $\geq 24 \mathrm{~h}$ after Tabata training in rats [46]. This transcriptional coactivator activates the transcription of proteins that have physiological functions, and its activity remained high for several days. When the human subjects exercised for 2 days, a high level of PGC1 $\alpha$ was maintained for half a week. This might explain why only 2 days of Tabata training enhanced the subjects' oxidative metabolism, in which dozens of enzyme proteins are involved in skeletal muscle.

In terms of the number of training sessions per day, the PGC1 $\alpha$ expression after 5 days of Tabata training performed $1 \times /$ day or $2 \times /$ day was compared in rats, and no difference was observed between these training groups. Although this finding was not from a human study, the results of other experiments suggest that the PGC $\alpha$ expression is saturated after Tabata training in rats (Terada and Tabata, unpublished observation). A further expression of PGC1 $\alpha$ and a subsequent increase in proteins that are transcriptionally stimulated by PGC1 $\alpha$ should thus not be expected when a human does additional Tabata training on the same day.

\section{Changes in muscle buffer capacity in muscle after Tabata training}

One of the most important changes explaining the improvement of the MAOD after HIIT-probably including Tabata training - is the enhanced buffer capacity of muscles recruited during the HIIT [56]. This enhanced capacity allows more muscle lactate formation, which results in proportional glycolytic ATP production for high-intensity exercises. Shark et al. reported that, after 8-week sprint training, their subjects' muscle buffer capacity was increased by $\sim 37 \%$. This robust increase in buffering capacity may explain the majority of the elevation of the MAOD after HIIIT including Tabata training.

In addition, carnosine is regarded as a minor contributing factor (5-10\%) to muscle buffer capacity [57]. In this context, it is interesting that the levels of the mRNA and protein of carnosine synthase 1 were increased by Tabata training [44], suggesting that the body's carnosine content might be elevated by Tabata training as demonstrated after a HIIT [58].

\section{The effects of Tabata training on circulation}

Endurance training increases capillary density [59]. Cocks et al. [60] reported that SIT and endurance training are equally effective at increasing skeletal muscle capillarization and the body's endothelial nitric oxide synthase (eNOS) content and at decreasing aortic stiffness. Using an animal model of Tabata training, Hasegawa et al. [61] observed that Tabata training decreased central arterial stiffness (assessed by arterial pulse wave velocity) to the same level as conventional aerobic training through the same arterial signal mechanism. This investigation showed that both animal 
model of Tabata training and aerobic training induced increased expression of eNOS, which produces nitric oxide (NOx), which subsequently may dilate arteries, suggesting that Tabata training might improve arterial function via the same mechanism as conventional aerobic training and may decrease the risk of cardiovascular events.

\section{The effects of Tabata training exercise on excess post-exercise oxygen consumption (EPOC) and diet-induced thermogenesis (DIT)}

There has been a rumor that Tabata training is effective for losing body weight. However, the energy consumption during a high-intensity short-duration training exercise is negligible. After the exercise, the body's oxygen uptake is higher than the resting metabolic rate, but EPOC after Tabata training exercise has not yet been quantified. The thermic effects of meals ingested after Tabata training have also not been evaluated. The resting oxygen uptake of human subjects after sprint-interval exercise was investigated with the use of a metabolic chamber for a $>22$-h period, including the subjects' intake of three meals. There were no observable differences in total resting oxygen uptake during the late recovery phase (3-22 h after the exercise period, at which point the EPOC had worn off) between the subjects' exercise and non-exercise control days, suggesting that there was no effect of the preceding SIT on the subsequent meal-induced thermogenesis [62].

In contrast, a recent investigation reported that oxygen uptake after lunch and supper ingested $1.5 \mathrm{~h}$ and $7.5 \mathrm{~h}$ after Tabata training exercise, respectively, was higher than that measured on the non- exercise day for subjects weighing $64.4 \pm 6.0 \mathrm{~kg}$ [63]. This study showed that EPOC during the first $1.5 \mathrm{~h}$ after the Tabata training exercise, and $\Delta$ DIT defined as a difference in resting oxygen uptake from 12:00-23:00 between Tabata training exercise and non-exercise control day are $115.3 \pm 32.3$ and $146.1 \pm 90.9 \mathrm{ml} \mathrm{kg}^{-1}$, respectively. These data suggest that EPOC and $\triangle D I T$ after Tabata training exercise were comparable with oxygen uptake during the Tabata training exercise $\left(123.4 \pm 12.0 \mathrm{ml} \mathrm{kg}^{-1}\right)$. Energy consumption calculated from oxygen uptake during 10-min warm up exercise (WU), Tabata training exercise was $74.3 \pm 5.2,39.8 \pm 6.3 \mathrm{kcal}$, respectively. EPOC during the first $1.5 \mathrm{~h}$ after Tabata training exercise and $\Delta \mathrm{DIT}$ after Tabata training are $37.5 \pm 12.7$, and $47.8 \pm 32.0 \mathrm{kcal}$, respectively. Summation of the previously described 4 energy consumption are $199.4 \pm 12.4 \mathrm{kcal}$ for the subjects. This value that is regarded as elevated energy consumption by Tabata training exercise could be the lowest in terms of securing weight reduction. However, after 6-week Tabata training, body weight was not changed [13]. Therefore, weight reducing effect of Tabata training seems to be minimal.
The Tsuji et al. study [63] also reported that the $\Delta$ DIT was correlated with the $\mathrm{VO}_{2}$ max of their subjects $\left(52.1 \pm 6.6 \mathrm{ml} \mathrm{kg}^{-1} \mathrm{~min}^{-1}\right)(r=0.76, n=10, p<0.05)$, suggesting that an improvement of the $\mathrm{VO}_{2}$ max may further enhance the energy consumption elevated by diet. Another recent investigation [64] demonstrated that the lunchenhanced thermogenesis ( $\triangle$ DIT: approx. $1.5-5.5 \mathrm{~h}$ after exercise for subjects weighing $67.9 \pm 7.7 \mathrm{~kg}$ ) after Tabata exercise $(15.7 \pm 10.4 \mathrm{kcal})$ was greater than that after $30 \mathrm{~min}$ of exercise at $70 \% \mathrm{VO}_{2} \max (6.6 \pm 8.4 \mathrm{kcal})$, suggesting that the $\Delta$ DIT of high-intensity exercise was greater than that of the moderate-intensity exercise. However, again, in terms of reducing body weight, the $\Delta$ DIT after high-intensity intermittent exercise is limited even when the $\Delta$ DIT after the consumption of a meal is considered.

\section{Recommended practical procedures for Tabata training}

The first article regarding Tabata training was published over 30 years ago, and no further paper was published by the authors of the original article until recently. There has thus been some confusion about Tabata training, especially concerning the methodology. The following practical tips for executing authentic Tabata training are presented in order to prevent the misunderstanding of Tabata training in future studies.

First, before an individual engages in Tabata training, warming up for $10 \mathrm{~min}$ at approx. $50 \% \mathrm{VO}_{2} \mathrm{max}$ is recommended $[12,13]$.

Authentic Tabata training consists of 7-8 exhaustive sets of 20-s high-intensity bicycle exercise (intensity: $170 \%$ $\left.\mathrm{VO}_{2} \max \right)$ with a 10 -s rest between the exercise bouts $[9,10]$. For determining the optimal exercise intensity of the training, the exercise intensity equivalent to the subject's $170 \%$ $V \mathrm{O}_{2} \max$ is first determined. The $170 \% \mathrm{VO}_{2} \max$ is an intensity that exhausts the subject by approx. $50 \mathrm{~s}$ of bicycling (if the subject continues to bicycle at that time) (Tabata, unpubl. data). The exercise intensity should be determined individually. The subject should then be instructed to continue bicycling until exhaustion (described below) after a 20 -s cycling bout with a 10-s rest interval. If the subject can continue to bicycle for more than eight sets, the exercise intensity should be increased. If the subject cannot bike for less than six sets, the exercise intensity is reduced. Therefore, the intensity of Tabata training does not have to be $170 \% \mathrm{VO}_{2} \max$; the intensity that exhausts the subject during the seventh or eighth set should be used.

Exhaustion during bicycle exercise is determined as follows. During the bicycling, when the pedaling frequency tends to less than the fixed rate [normally 90 repetitions per minute (rpm)], we encourage the subject verbally with phrases such as 'Come on, come on!' With this verbal 
encouragement, a subject can often increase the pedaling frequency to $90 \mathrm{rpm}$. When encouragement has been given but the subject's pedaling frequency gradually declines to $85 \mathrm{rpm}$, we define it as exhaustion and let the subject stop bicycling.

For bicycling exercise, it is important to raise the pedaling frequency to the fixed rate as soon as possible to set the correct load for the subject. A pedaling frequency of $100 \mathrm{rpm}$ might be good for cyclists. In the original Tabata study, $90 \mathrm{rpm}$ was used. The reason why we use a higher pedaling frequency than that used for normal bicycle exercise (50-70 rpm) is that without such a high pedaling frequency, a high-enough load cannot be set for heavyweight top athletes. In the original Tabata training experiments, Monark bicycles (whose highest load is $7 \mathrm{kP}$ ) were used, but even with such a heavy weight, the load is not high enough to exhaust elite athletes within 7-8 sets of Tabata training if 50-70 rpm is used. By using $90 \mathrm{rpm}$, an adequate work rate, which is a function of weight (kP) and $\mathrm{rpm}$, is assured for highly trained athletes.

\section{Possible adverse effects of Tabata training}

Because high-intensity exercise may reduce immunological functions [65], it can be speculated that in terms of the prevention of any type of cancer that may be initiated by low immunological function, high-intensity exercise training may have no effects or adverse effects. However, the results of a recent study suggested that Tabata training may help prevent colon cancer by enhancing the secretion and elevating the blood concentration of secreted protein acidic and rich in cysteine (SPARC) (Fig. 9), a myokine that decreases the number of aberrant crypt foci (ACF), which is the first step of colon cancer induction, by inducing the apoptosis of ACF in the colon [66]. These biological results may explain the epidemiological finding that vigorous exercise may help prevent and not worsen colon cancer [67, 68]. Another recent investigation indicated that this result may reflect the evidence that Tabata training does not reduce immunological function [69].

\section{Important contributions of other scientists}

Among the many scientists who have examined Tabata training and other HIIT methods, the contribution of Dr. Martin Gibala to the popularity of HIIT among scientists and exercisers should be appreciated [52, 70-72]. He and his team conducted numerous studies on skeletal muscle adaptation after HIIT. These studies helped scientists further understand HIIT including Tabata training.

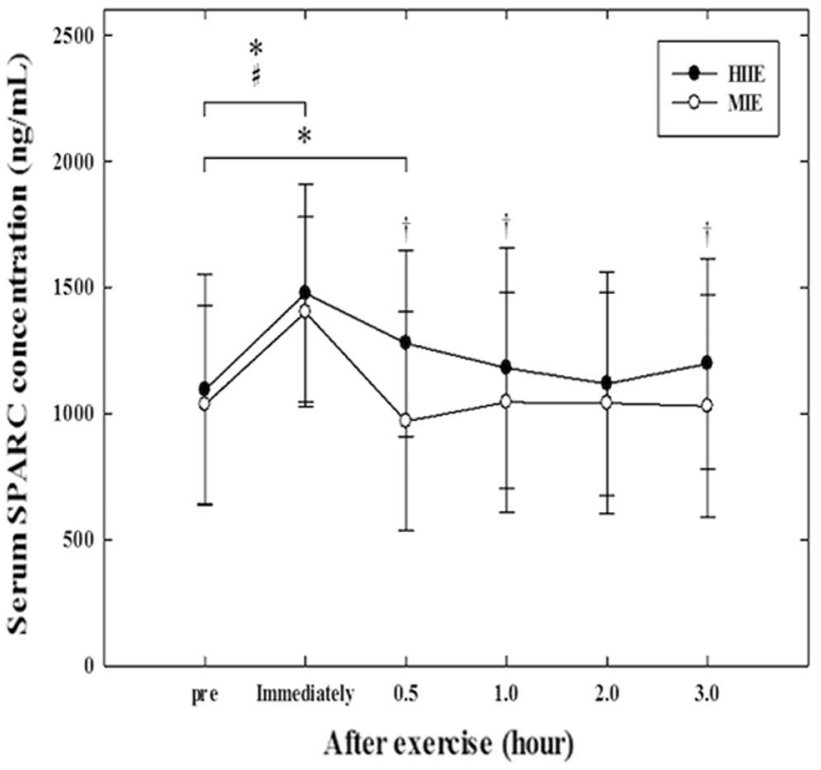

Fig. 9 Effects of HIIE ( $\bullet$ : Tabata training exercise) and 30-min moderate-intensity ( $70 \% \mathrm{VO}_{2} \mathrm{max}$ ) prolonged exercise (MIE) $(\mathrm{O})$ on the serum SPARC concentration in human subjects [66]. Values are mean \pm SD. $* p<0.05$ vs. the pre-exercise values of the HIIE experiments. ${ }^{\#} p<0.05$ vs. the pre-exercise values of the MIE. ${ }^{\dagger} p<0.05$ between the HIIE and MIE at the same time points

\section{Future research}

The use of Tabata training is usually an individual attempt by coaches and/or athletes. Scientific evidence from the sport fields is thus limited. Ravier reported that a running training protocol that was similar to Tabata training elevated the $V \mathrm{O}_{2}$ max and MAOD of karate athletes who also performed other types of repetitive exercise [73]. Evaluations of top athletes by researchers will help elucidate the effects of Tabata training on the performance of various sports. A determination of the effects of Tabata training using exercises that simulate the activities of specific sports is valuable in light of the specificity of training and training effects on sports performance.

However, in terms of effects on aerobic and anaerobic energy-releasing systems, there is not enough published data about training methods that follow protocols similar to Tabata training but use other types of exercise including running and various body-weight bearing exercises, which are frequently adopted by competitive runners and recreational exercisers, respectively. Effects of such exercise training on $\mathrm{VO}_{2}$ max have been reported [74-80], and such research on similar protocol training methods is expected to determine the effects on anaerobic energy-releasing systems.

Since Tabata training induces the expression of proteins related not only to sports performance but also to health promotion [44], more research on the possible effects of Tabata 
training and other training that uses a Tabata protocol on health outcomes is expected.

Tabata training is very demanding [33], and thus participation in Tabata training might be limited to highly motivated athletes who are familiar with the scientific evidence regarding Tabata training or are persuaded to engage in the training by coaches who know the Tabata training research findings. In a study of women who were simply recreationally active, their perceived enjoyment of a weight-bearing HIIT increased from pre- to post-training, suggesting that chronic exposure to such training may elevate people's enjoyment of the training [78]. In addition, the dropout rate in the Chuiesiri et al. study of obese pre-adolescent boys [80] was quite low (6.3\%), and Logan et al. reported a high adherence rate among inactive volunteer adolescents to an all-out-type HIIT using various types of weight bearing exercise; $90 \%$ of their subjects completed the regimen [79]. These rates may indicate that the HIIT was tolerable and positively accepted. However, the results of another investigation suggested that training at high intensities would be rated as not enjoyable [81]. A psychological inquiry regarding study subjects' enjoyment of Tabata training is required. The development of low-intensity training using a Tabata protocol and training at the same intensity as that used in Tabata training (7-8 sets) but with a smaller number (3-4 sets) of exercise bouts [82] is expected; with a lower-intensity protocol, subjects would more easily enjoy the training.

As noted above, it also is necessary to investigate the possible detrimental side effects of Tabata training and other types of HIIT and to find solutions to prevent such side effects by diet/supplements, other physical conditioning, and/or other methods.

Lastly, in order to prescribe science-based training, more basic research on HIIT involving Tabata training is required to further delineate the mechanisms underlying the beneficial effects of this training on both sport-oriented and healthoriented outcomes, both of which contribute to improved quality of life.

\section{Conclusions}

Our studies have demonstrated that 6- to 12-week Tabata training increases the body's $V_{2}$ max by $9.2-15.0 \%$ and the MAOD by $20.9-35.0 \%$ [13, 36, 44]. A 2018 review of the Tabata protocol, which included various body-weightbearing exercises, indicates that the $\mathrm{VO}_{2}$ max is elevated by 5-18\% after the training lasting 4-12 weeks [83]. The magnitude of the elevation of the $V \mathrm{O}_{2}$ max after Tabata training is similar to those of other HIIT and moderate-intensity aerobic exercise training types [83]. 6-week Tabata training increased the MAOD by $17-28 \%$ [13, 36, 44]. These values are comparable to those obtained by other HIIT types [74, 75].

In conclusion, these improvements of both the aerobic and anaerobic energy-releasing systems after Tabata training are comparable to those provided by conventional aerobic and anaerobic training, including other types of HIIT, suggesting that Tabata training is useful to enhance sports performances that depend on both the aerobic and anaerobic energy-releasing systems for resynthesizing the ATP used during the specific sports.

\section{References}

1. Fox EL, Mathews DK (1974) Interval training: conditioning for sports and general fitness. WB. Saunders, Philadelphia

2. (https://en.wikipedia.org/wiki/Fartlek). Accessed 9 Feb 2019

3. Kenny WL, Wilmore JH, Costill DL (2012) Physiology of sport and exercise, 5th edn. Human Kinetics, Champaign

4. Thoma Z (1982) Život Emila Zátopka. Translated into Japanese by Otake K. Baseball Magazine Sha, Tokyo

5. Fox EL (1979) Sport physiology. Saunders, Philadelphia

6. Fox EL, Bartels RL, Billings CE, Mathews DK, Bason R, Webb WH (1973) Intensity and distance of interval training programs and changes in aerobic power. Med Sci Sports Exerc 5:18-22

7. Fox EL, Bartels RL, Billings CE, O’Brien R, Bason R, Mathews DK (1975) Frequency and duration of interval training programs and changes in aerobic power. J App Physiol 38:481-484

8. Garber CE, Blissmer B, Deschenes MR, Franklin BA, Lamonte MJ, Lee IM, Nieman DC, Swain DP (2011) Quantity and quality of exercise for developing and maintaining cardiorespiratory, musculoskeletal, and neuromotor fitness in apparently healthy adults: guidance for prescribing exercise. Med Sci Sports Exerc 43:1334-1359

9. Hermansen L, Medbø JI, Mohn A-C, Tabata I, Bahr R (1984) Oxygen deficit during maximal exercise of short duration. Acta Physiol Scand 121:39A

10. Medbø JI, Mohn A-C, Tabata I, Bahr R, Vaage O, Sejersted OM (1988) Anaerobic capacity determined by maximal accumulated $\mathrm{O}_{2}$ deficit. J Appl Physiol 64:50-60

11. Krogh A, Lindhard J (1920) The changes in respiration at the transition from work to rest. J Physiol 53:431-437

12. Tabata I, Irisawa K, Kouzaki M, Nishimura K, Ogita F, Miyachi M (1997) Metabolic profile of high-intensity intermittent exercises. Med Sci Sports Exerc 29:390-395

13. Tabata I, Nishimura K, Kouzaki M, Hirai Y, Ogita F, Miyachi M, Yamamoto K (1996) Effects of moderate intensity-endurance and high intensity-intermittent training on anaerobic capacity and $V_{\mathrm{O}_{2}} \mathrm{max}$. Med Sci Sports Exerc 28:1327-1330

14. https://www.merriam-webster.com/dictionary/interval. Accessed 9 Feb 2019

15. https://www.merriam-webster.com/dictionary/intermittent. Accessed 9 Feb 2019

16. Weston KS, Wisløff U, Coombes JS (2014) High-intensity interval training in patients with lifestyle-induced cardiometabolic disease: a systematic review and meta-analysis. Br J Sports Med 48(16): 1227-1234

17. Thompson WE (2017) Worldwide survey of fitness trends for 2018: the CREP edition. ACSM's Health Fit 21(6):10-19

18. https://www.merriam-webster.com/dictionary/sprint. Accessed 9 Feb 2019 
19. Sloth M, Sloth K, Overgaard Dalgas U (2013) Effects of sprint interval training on $\mathrm{VO}_{2} \max$ and aerobic exercise performance: a systematic review and meta-analysis. Scand J Med Sci Sports 23:e341-e352

20. Gist NH, Fedewa MV, Dishman RK, Cureton KJ (2014) Sprint interval training effects on aerobic capacity: a systematic review and meta-analysis. Sports Med 44:269-279

21. Nevill AM, Ramsbottom R, Williams C (1992) Scaling physiological measurements for individuals of different body size. Eur J Appl Physiol Occup Physiol 65:110-117

22. Ramsbottom R, Nevill AM, Nevill ME, Newport S, Williams C (1994) Accumulated oxygen deficit and short-distance running performance. J Sports Sci 12:447-453

23. Robinson D, Harmon PM (1941) The effects of training and gelatin upon certain factors which limits muscular work. Am J Physiol 133:161-169

24. Knehr CA, Dill DB, Neufeld W (1942) Training and its effects on man at rest and at work. Am J Phyiol 136:148-156

25. Hickson RC, Bomze HA, Holloszy JO (1977) Linear increase in aerobic power induced by a strenuous program of endurance exercise. J Appl Physiol Respir Environ Exerc Physiol 42:372-376

26. Medb $\varnothing$ JI, Burgers S (1990) Effect of training on the anaerobic capacity. Med Sci Sports Exerc 22:501-507

27. Astrand P-O, Rodahl K (1977) Textbook of work physiology: Physiological bases of exercise, 2nd edn. McGraw, New York

28. Medbø JI, Tabata I (1989) Relative importance of aerobic and anaerobic energy release during short-lasting exhaustive bicycle exercise. J Appl Physiol 67:1881-1886

29. Taylor HL, Buskirk E, Henshel A (1955) Maximal oxygen intake as an objective measure of cardiorespiratory performance. J Appl Physiol 8:73-80

30. Noordhof DA, Vink AM, de Koning JJ, Foster C (2011) Anaerobic capacity: effect of computational method. Int J Sports Med 32(6):422-428

31. Ogita F, Hara M, Tabata I (1996) Anaerobic capacity and maximal oxygen uptake during arm stroke, leg kicking and whole body swimming. Acta Physiol Scand 157:435-441

32. Viana RB, Navesa JPA, de Liraa CAB, Coswigb VS, Del Vecchioc FB, Vieiraa CA, Gentila P (2018) Defining the number of bouts and oxygen uptake during the "Tabata protocol" performed at different intensities. Physiol Behav 189:10-15

33. Foster C, Farland CV, Guidotti F, Harbin M, Roberts B, Schuette J, Tuuri A, Doberstein ST, Porcari JP (2015) The effects of high-intensity interval training vs steady state training on aerobic and anaerobic capacity. J Sports Sci Med 14:747-755

34. Kouzaki M, Tabata I (1998) Effects of high-intensity intermittent training on maximal oxygen deficit and maxima oxygen uptake. J Train Sci 9:83-94 (in Japanese)

35. Ogita F, Huang Z, Kurobe K, Ozawa G, Nagira A, Yotani K, Taguchi N, Tamaki H (2014) Effects of sprint interval training on metabolic, mechanical characteristics and swimming performance. In: Proceedings of the 12th international symposium on biomechanics and medicine in swimming. Mason B, Barners D, Jukes D, Vlahovich N, eds. The Australian Institute of Sport, Canberra, Australia: 453-457

36. Hirai Y, Tabata I (1996) Effect of high-intensity intermittent training and resistance training on the maximal oxygen deficit and $\mathrm{VO}_{2}$ max. Jpn J Phys Fit Sport Med 45:495-502 (in Japanese)

37. Minahan C, Wood C (2008) Strength training improves supramaximal cycling but not anaerobic capacity. Eur J Appl Physiol 102:659-666

38. Hickson RC (1980) Interference of strength development by simultaneously training for strength and endurance. Eur J Appl Physiol 45:255-263
39. Bergström J, Hultman E (1966) Muscle glycogen synthesis after exercise: an enhancing factor localized to the muscle cells in man. Nature 210:309-310

40. Gollnick P, Armstrong RB, Saltin B, Saubert IVCW, Sembrowich WL, Shephard RE (1973) Effect of training on enzyme activity and fiber composition of human skeletal muscle. J Appl Phsyiol 34:107-111

41. Holloszy JO, Oscai LB, Mole PA, Don IJ (1971) Biochemical adaptations to endurance exercise in skeletal muscle. Muscle metabolism during exercise. In: Pernow B, Saltin B (eds) Advances in experimental medicine and biology, vol 11. Plenum Press, New York

42. Yamaguchi W, Fujimoto E, Higuchi M, Tabata I (2011) A DIGE proteomics analysis for low-intensity exercise-trained rat skeletal muscle. Jpn J Phys Fit Sports Med 60:511-518 (in Japanese)

43. Thorstensson A, Sjödin B, Karlsson J (1975) Enzyme activities and muscle strength after "sprint training" in man. Acta Physiol Scand 94:313-318

44. Miyamoto-Mikami E, Tsuji K, Horii N, Hasegawa N, Fujie S, Homma T, Uchida M, Hamaoka T, Kanehisa H, Tabata I, Iemitsu M (2018) Gene expression profile of muscle adaptation to highintensity interval training in young men. Sci Rep 8:16811

45. Terada S, Tabata I, Higuchi M (2004) Effect of high-intensity intermittent swimming training on fatty acid oxidation enzyme activity in rat skeletal muscle. Jpn J Physiol 54:47-52

46. Terada S, Kawanaka K, Goto M, Shimokawa T, Tabata I (2005) Effects of high-intensity intermittent swimming on PGC-1 $\alpha$ protein expression in rat skeletal muscle. Acta Physiol Scand 184:59-65

47. Wu Z, Puigserver P, Andersson U, Zhang C, Adelmant G, Mootha V, Troy A, Cinti S, Lowell B, Scarpulla RC, Spiegelman BM (1999) Mechanisms controlling mitochondrial biogenesis and respiration through the thermogenic coactivator PGC1. Cell 98:115-124

48. Terada S, Yokozeki T, Kawanaka K, Ogawa K, Higuchi M, Ezaki O, Tabata I (2001) Effects of high-intensity intermittent swimming training on GLUT-4 and glucose transport activity in rat skeletal muscle. J Appl Physiol 90:2019-2024

49. Yamaguchi W, Fujimoto E, Higuchi M, Tabata I (2010) A DIGE proteomic analysis for high-intensity exercise-trained rat skeletal muscle. J Biochem (Tokyo) 148:327-333

50. MacInnis MJ, Gibala MJ (2017) Physiological adaptations to interval training and the role of exercise intensity. J Physiol 595:2915-2930

51. Robinson MM, Dasari S, Konopka AR, Johnson ML, Manjunatha S, Esponda RR, Carter RE, Lanza IR, Nair KS (2017) Enhanced protein translation underlies improved metabolic and physical adaptations to different exercise training modes in young and old humans. Cell Metab 25:581-592

52. Burgomaster KA, Hughes SC, Heigenhauser GJ, Bradwell SN, Gibala M (2005) Six session of sprint interval training increases muscle oxidative potentials and cycle endurance capacity in humans. J Appl Physiol 98:1985-1990

53. Daussin FN, Zoll J, Dufour SP, Ponsot F, Lonsdorfer-Wolf E, Doutreleau S, Mettauer B, Piquard F, Geny B, Richard R (2008) Effect of interval versus continuous training on cardiorespiratory and mitochondrial functions: relationship to aerobic performance improvement in sedentary subjects. Am J Phsyiol Regul Integr Comp Physiol 295:R264-R272

54. Macpherson RE, Hazell TJ, Olver TD, Paterson DH, Lemon PW (2011) Run sprint interval training improves aerobic performance but not maximal cardiac output. Med Sci Sports Exerc 43:115-122

55. Astorino TA, Edmunds RM, Clark A, King L, Gallant RA, Namm S, Fischer A, Wood KM (2017) High-intensity interval training 
increases cardiac output and $\mathrm{VO}_{2} \mathrm{max}$. Med Sci Sports Exerc 49:265-273

56. Sharp RL, Costill DL, Fink WJ, King DS (1986) Effects of eight weeks of bicycle ergometer sprint training on human muscle buffer capacity. Inter J Sports Med 7:13-17

57. Sahlin K (2014) Muscle energetics during explosive activities and potential effects of nutrition and training. Sports Med 44(Suppl e):S167-S173

58. De Salles Painelli V, Nemezio KM, Pinto AJ, Franchi M, Andrade I, Riani LA, Saunders B, Sale C C, Harris RC, Gualano B, Artioli GG (2018) High-intensity interval training augments muscle carnosine in the absence of dietary beta-alanine intake. Med Sci Sports Exerc 50:2242-2252

59. Hermansen L, Wachtlova M (1971) Capillary density of skeletal muscle in well-trained and untrained men. J Appl Physiol 30:860-863

60. Cocks M, Shaw CS, Shepherd SO, Fisher JP, Ranasinghe A, Barker TA, Wagenmakers JM (2016) Sprint interval and moderate-intensity continuous training have equal benefits on aerobic capacity, insulin sensitivity, muscle capillarisation and endothelial eNOS/NAD(P)Hoxidase protein ratio in obese men. J Physiol 594:2307-2321

61. Hasegawa N, Fujie S, Horii N, Miyamoto-Mikami E, Tsuji K, Uchida M, Hamaoka T, Tabata I, Iemitsu M (2018) Effects of different exercise models on arterial stiffness and nitric oxide synthesis. Med Sci Sports Exerc 50(6):1177-1185

62. Sevits KJ, Melanson EL, Swibas T, Binns SE, Klochak AL, Lonac MC, Peltonen GL, Scalzo RL, Schweder MM, Smith AM, Wood LM, Melby CL, Bell C (2013) Total daily energy expenditure is increased following a single bout of sprint interval training. Physiol Rep 1:e00131

63. Tsuji K, Xu Y, Liu X, Tabata I (2017) Effects of short-lasting supramaximal-intensity exercise on diet-induced increase in oxygen uptake. Physiol Rep 5:e13506

64. Tsuji K, Xu Y, Tabata I (2019) Effects of moderate-intensity exercise on diet-induced increase in resting oxygen uptake. J Phys Fit Sports Med 8:15-27

65. Nieman DC (1994) Exercise, upper respiratory tract infection, and the immune system. Med Sci Sport Exerc 26:128-139

66. Matsuo K, Sato K, Suemoto K, Miyamoto-Mikami E, Fuku N, Higashida K, Tsuji K, Xu Y, Liu X, Iemitsu M, Hamaoka T, Tabata I (2017) A mechanism underlying preventive effect of high-intensity training on colon cancer. Med Sci Sports Exerc 49:1805-1816

67. Kato I, Tominaga S, Ikari A (1990) A case-control study of male colorectal cancer in Aichi prefecture, Japan: with special reference to occupational activity level, drinking habits and family history. Jpn J Cancer Res 81:115-121

68. Kono S, Shinchi K, Ikeda N, Yanai F, Imanishi K (1991) Physical activity, dietary habits and adenomatous polyps of the sigmoid colon: a study of self-defense officials in Japan. J Clin Epidemiol 44:1255-1261

69. Harnish CR, Sabo RT (2016) Comparison of two different sprint interval training work-to-rest ratios on acute inflammatory responses. Sports Med 2:20
70. Gibala MJ, Hawley JA (2017) Sprinting toward fitness. Cell Metab 25:989-990

71. Gillen JB, Martin MJ, MacInnis MJ, Skelly LE, Tarnopolsky MA, Gibala MJ (2016) Twelve weeks of sprint interval training improves indices of cardiometabolic health similar to traditional endurance training despite a five-fold lower exercise volume and time commitment. PLoS One 11:e0154075

72. MacInnis MJ, Gibala MJ (2017) Physiological adaptations to interval training and the role of exercise intensity. J Physiol 595:2915-2930

73. Ravier G, Dugue B, Grappe F, Rouillon JD (2009) Impressive anaerobic adaptations in elite karate athletes due to few intensive intermittent sessions added to regular karate training. Scand J Med Sci Sports 19:687-694

74. Ramsbottom R, Nevill AM, Seager RD, Hazeldine R (2001) Effect of training on accumulated oxygen deficit and shuttle run performance. J Sports Med Phys Fit 41:281-282

75. Weber CL, Schneider DA (2002) Increases in maximal accumulated oxygen deficit after high-intensity interval training are not gender dependent. J Appl Physiol 92:1795-1801

76. Fortner HA, Salgado JM, Holmstrup AM, Hosmstrup ME (2014) Cardiovascular and metabolic demands of the kettlebell swing using Tabata interval versus a traditional resistance protocol. Int J Exerc Sci 7:179-185

77. McRae G, Payne A, Zelt JGE, Scribbans TD, Jung ME, Little JP, Gurd BJ (2012) Extremely low volume, whole-body aerobic resistance training improves aerobic fitness and muscular endurance in females. Appl Physiol Nutr Metab 37:1124-1131

78. Funch LT, Lind E, True L, Van Langen D, Foley JT, Hokanso JF (2017) Four weeks of off-season training improves peak oxygen consumption in female field hockey players. Sports 5:89

79. Logan GRM, Harris N, Duncan S, Planks LD, Merien F, Schofields G (2016) Low-active male adolescents: a dose response to high-intensity interval training. Med Sci Sports Exerc 48:481-490

80. Chuiesiri N, Suksom D, Tanaka H (2018) Effects of high-intensity intermittent training on vascular function in obese preadolescent boys. Child Obes 14:41-49

81. Ekkekakis P, Hall EE, Petruzzello SJ (2008) The relationship between exercise intensity and affective responses demystified: to crack the 40-year-old nut, replace the 40-year-old nutcracker! Ann Behav Med. 35:136-149

82. Fujimoto E, Machida S, Higuchi M, Tabata I (2010) Effects of nonexhaustive bouts of high-intensity intermittent swimming training on GLUT-4 expression in rat skeletal muscle. J Physiol Sci 60:95-101

83. Viana RB, Barbosa de Lira CA, Naves JPA, Coswig VS, Del Vecchio FB, Gentil P (2019) Tabata protocol: a review of its application, variations and outcomes. Clin Physiol Funct Imaging 39:1-8

Publisher's Note Springer Nature remains neutral with regard to jurisdictional claims in published maps and institutional affiliations. 